\title{
Contribución a la micobiota liquénica del bosque seco tropical colombiano (Montes de María, Sucre)
}

\section{Contribution to lichen micobiota of the colombian tropical dry forest (Montes de María, Sucre)}

\author{
Steven García-Martínez ${ }^{\mathrm{a}}$ \\ Jorge D. Mercado-Gómez
}

Fecha de Recepción: 12.02.2020

Fecha de Aceptación: 05.05.2020

Doi: https://doi.org/10.19053/01217488.v11.n2.2020.11000

\begin{abstract}
Resumen
Se presenta el primer inventario de especies de hongos liquenizados encontrados en fragmentos de bosque seco tropical en los Montes de María, departamento de Sucre. Con el fin de determinar la composición de líquenes en el área de estudio, se realizaron muestreos a través de transectos lineales $(100$ x $10 \mathrm{~m})$ en seis localidades: Colosó, Chalán, Toluviejo, Morroa, Ovejas y San Onofre. Para identificar si las especies correspondían a nuevos registros corológicos, datos sobre su distribución geográfica fueron analizados a partir de literatura especializada. Un total de 93 especies, distribuidas en 40 géneros y 21 familias. Todas las especies corresponden a nuevos reportes para el departamento de Sucre y 22 para Colombia. De esta forma, en el presente estudio se hace una contribución al conocimiento de la biota liquénica de bosques secos tropicales colombianos.
\end{abstract}

Palabras clave: corología, composición, líquenes, riqueza.

\begin{abstract}
We present the first inventory of lichenized fungi found in tropical dry forest fragments in Montes de María, department of Sucre. Through linear transects $(100 \times 10 \mathrm{~m})$, we surveyed six localities: Colosó, Chalán, Toluviejo, Morroa, Ovejas, and San Onofre. In order to identify whether species represented new chorological records, geographic distribution data were obtained from specialized literature. A total of 93 species distributed in 40 genera and 21 families were identified. All species are new records for the department of Sucre and 22 for Colombia. In this way, we made a contribution to the knowledge of the lichen biota of the Colombian tropical dry forest.
\end{abstract}

Keywords: chorology, composition, lichens, richness.

\footnotetext{
a Grupo Evolución y Sistemática Tropical. Universidad de Sucre, Carrera 28 Nº. 5-27. Sincelejo, Colombia. stgarciamtz@gmail.com

b Grupo Evolución y Sistemática Tropical. Departamento de Biología y Química. Universidad de Sucre, Carrera 28 №. 5-27. Sincelejo, Colombia. jorge.mercado@unisucre.edu.co
} 


\section{INTRODUCCIÓN}

El levantamiento de los Andes ha sido uno de los principales eventos formadores de diversidad biológica en Sur América [1]. En Colombia, por ejemplo, la formación de esta cadena montañosa produjo profundos cambios en la topografía y condiciones climáticas que generaron múltiples oportunidades para la adaptación y diversificación de especies [1]. En este sentido, históricamente se ha considerado que diferentes grupos taxonómicos como los briofitos, helechos, líquenes, entre otros, presentan una alta diversidad en los bosques Andinos, lo cual ha llevado a que estudios sobre riqueza, taxonomía, ecología y biogeografía se hayan enfocado principalmente en los Andes [2-8]. Sin embargo, poco es conocido sobre la diversidad de estos taxones en otras regiones [9]. En el Caribe colombiano, por ejemplo, aunque recientemente se han incrementado el estado del conocimiento taxonómico y ecológico de grupos biológicos como los líquenes [10-19], aún existen diferentes zonas o biomas aun sin explorar.

Los bosques secos tropicales son formaciones vegetales que se distribuyen de forma discontinua desde el sur de México hasta el Norte de Argentina, incluyendo las Antillas [20]. Estas formaciones ocurren en sitios con temperaturas por encima de $\operatorname{los} 25^{\circ} \mathrm{C}$, precipitaciones anuales entre 700 a $2000 \mathrm{~mm}$ anuales y presentan entre dos o más de tres meses donde las precipitaciones son inferiores a los $1000 \mathrm{~mm}$ Antillas [20]. En Colombia, estos bosques se distribuyen principalmente en los valles de los ríos Magdalena y Valle, pero también en las planicies del Caribe [21]. Sobre esta región se encuentran los Montes de María, considerado como uno de los fragmentos de bosque seco tropical en mejor estado de conservación [21]. Sin embargo, aunque diferentes estudios han incrementado el conocimiento sobre la diversidad de la fauna y flora en esta región [22-26], poco es conocido sobre taxones como los líquenes, más aun cuando históricamente se ha asumido que la diversidad de plantas no vasculares era muy reducida para estos montes [9, 27]. Empero, en años recientes se ha podido establecer que la composición y diversidad de briofitos, por ejemplo, es mayor de la esperada, de tal forma que el número de registros se ha incrementado para los ecosistemas secos [28, 29]. Bajo este contexto, en la presente investigación se llevó a cabo una caracterización florística de líquenes en seis localidades en los Montes de María (departamento de Sucre), con el fin de conocer la riqueza de estos organismos en uno de los mejores fragmentos de bosque seco tropical de Colombia [21]. Además, enriquecer el conocimiento de estos organismos en los bosques secos y, por lo tanto, en Colombia, a través de la identificación de nuevos registros corológicos.

\section{MATERIALES Y MÉTODOS}

\section{Área de estudio}

Los Montes de María se encuentran localizados en el norte de Colombia, sobre las planicies del Caribe colombiano, en los departamentos de Sucre y Bolívar. En el departamento de Sucre, estos montes presentan una extensión de 6.297 $\mathrm{km}^{2}$ [30], dentro de las cuales se ha establecido la Reserva Forestal Protectora Serranía de Coraza y Montes de María [31-33]. Esta zona posee un rango de altitud que va desde los 0 hasta los 1000 $\mathrm{m}$, caracterizados principalmente por un relieve abrupto e irregular formado por montañas y colinas fuertemente escarpadas, de afloramientos rocosos y arcillolitas de origen marino. Los suelos presentes en la zona son originados a partir de mantos arenosos, arcillosos y de calizas del Mioceno [34].

El clima es bimodal, abarcando periodos húmedos y secos con temperaturas que oscilan entre los 26 y $30^{\circ} \mathrm{C}$ [30]. La precipitación media anual es de $1.500 \mathrm{~mm}$ y la humedad relativa varía entre $75 \%$ y $85 \%$, con incremento en el segundo semestre del año [31, 32]. En relación con la hidrología, el área de estudio se encuentra dentro de una red hidrográfica perteneciente a la cuenca del Golfo de Morrosquillo [35], la cual forma un sistema de microcuencas que da origen a numerosas quebradas y cuerpos de agua que tienden a desaparecer en la época de sequía, pero son abastecidos con los llamados "ojos de agua" formados en las montañas como depósitos de agua [30]. 


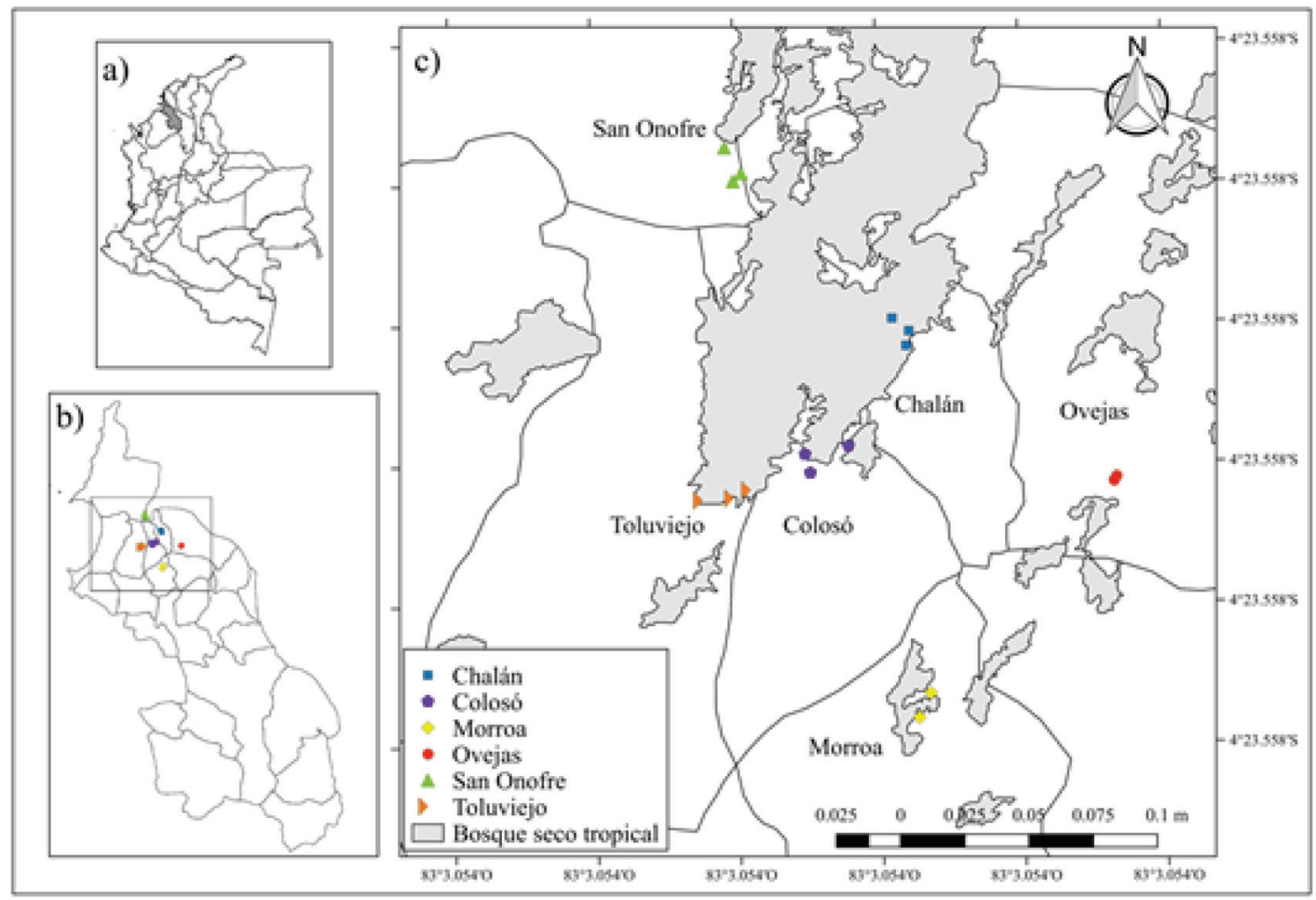

Figura 1. Localización geográfica del área de estudio en (a) Colombia, (b) el departamento de Sucre y (c) Montes de María, detallando el polígono de bosque seco tropical para Colombia.

En lo que a flora se refiere, la vegetación corresponde a bosque seco tropical y entre sus especies de árboles más importantes se encuentran Cynophalla sessilis (DC.) J. Presl, Ampelocera edentula Kuhlm., Aspidosperma polyneuron Müll. Arg., Brosimum alicastrum Sw., Myrcia fallax (Rich.) DC. y Simira cordifolia (Hook.f.) Steyerm. [36]. Cabe destacar que esta vegetación ha sido sometida a procesos de deforestación relacionados con actividades agrícola-ganaderas, lo cual induce a la pérdida de la continuidad de los relictos o fragmentos de bosques que aún existen en el área de estudio [37], problemática que conllevó a la creación de la Reserva Forestal Protectora Serranía de Coraza y Montes de María, ubicada en los municipios Colosó, Chalán y Toluviejo.

\section{Diseño de muestreo y recolecta de ejemplares}

Para seleccionar localidades para llevar a cabo los levantamientos de información biológica, se realizó un análisis fotointerpretativo de imágenes Landsat 8 ETM del servicio geológico de los Estados Unidos (USGS) con resolución espacial de 30 m (http://www.usgs. gov/). Luego se seleccionaron las áreas con mayor grado de vegetación donde se escogieron treinta sitios de muestreos; además, se consideró la accesibilidad y el orden público.
De esta forma, sobre fragmentos de bosque seco encontrados en los municipios de Colosó, Chalán, Toluviejo, Morroa, Ovejas y San Onofre (Figura 1) fue inventariada la flora liquénica a través de tres transectos lineales de $100 \times 10 \mathrm{~m} 2$.

\section{Determinación taxonómica}

El proceso de determinación taxonómica se realizó a través de claves y monografías taxonómicas, hasta género se emplearon a Sipman (2005) y Cáceres [45]. Para Graphidaceae Lücking, et al. [38] y Lücking and Rivas [39], en Lecanoraceae Lumbsch, et al. [40], Malmideaceae Breuss and Lücking [41], Parmeliaceae [42], Physciaceae Benatti and Marcelli [43], Pyrenulaceae Aptroot [44] y en Trypetheliaceae Aptroot and Lücking [45] y Aptroot, et al. [46]. La clasificación de las especies, se organizó según el sistema propuesto por Lücking, et al. [47]y la nomenclatura según Index Fungorum [http: //www.indexfungorum. org]. Además, se emplearon pruebas de reacción química, de acuerdo a los lineamientos propuestos por Chaparro and Aguirre [48]. El material determinado fue depositado en el herbario HEUS bajo la enumeración de García-M. Finalmente y con el objeto de determinar si las especies identificadas correspondían a nuevos registros corológicos para 
el Caribe Colombiano y para Sucre, se realizó una búsqueda detallada de los registros establecidos en los herbarios en línea de la Universidad Nacional (http://www.biovirtual.unal.edu.co/ICN/) y literatura especializada $[3,11,12,16,49]$.

\section{RESULTADOS}

Composición y riqueza taxonómica: Se identificaron 92 especies segregados en 40 géneros y 21 familias. Con base en la búsqueda de nuevos registros corológicos se pudieron identificar 22 reportes nuevos para el Colombia y 92 para el departamento de Sucre (Tabla 1). En términos de riqueza de especies, la familia Graphidaceae es la mejor representada con $44 \%$, seguida por Trypetheliaceae (8\%), Malmideaceae (7\%), Ramalinaceae (6\%) y Parmeliaceae (5\%). Arthoniaceae, Caliciaceae, Collemataceae y Lecanographaceae poseen un $3 \%$, el resto de familias poseen dos y una especie, las cuales en conjunto representan el 17\%. Los géneros más diversos fueron Graphis, Malmidea, Parmotrema, Ramalina, Astrothelium, Leptogium y Pyrenula. Los demás géneros estuvieron constituidos por menos de dos especies (Tabla 1).

Tabla 1. Listado de especies liquénicas y su distribución en áreas de muestreos en los Montes de María-Sucre. *Nuevo registro para el Caribe colombiano, **nuevo registro para Colombia. Todas las especies son nuevos registros para el departamento de Sucre. Acrónimos: Cl: Colosó, Ch: Chalán, Tv: Toluviejo, Mr: Morroa, Ov: Ovejas y So: San Onofre.

\begin{tabular}{|c|c|c|c|}
\hline Familias & Géneros & Especies & Localidades \\
\hline \multirow{3}{*}{ Arthoniaceae } & Coniocarpon & Coniocarpon cinnabarinum DC. & $\mathrm{Mr}, \mathrm{Tv}$ \\
\hline & \multirow{2}{*}{ Herpothallon } & Herpothallon minimum Aptroot \& Lücking & $\mathrm{Ch}$ \\
\hline & & Herpothallon rubrocinctum (Ehrenb.) Aptroot et al. & $\mathrm{Ch}$ \\
\hline \multirow{3}{*}{ Caliciaceae } & Dirinaria & Dirinaria picta (Sw.) Clem. \& Shear & $\mathrm{Cl}, \mathrm{Mr}, \mathrm{Ov}, \mathrm{So}, \mathrm{Tv}$ \\
\hline & \multirow{2}{*}{ Pyxine } & Pyxine sorediata (Ach.) Mont. * & $\mathrm{Cl}, \mathrm{Ov}$ \\
\hline & & Pyxine subcinerea Stirt. * & $\mathrm{Tv}$ \\
\hline \multirow{2}{*}{ Chrysothrichaceae } & \multirow{2}{*}{ Chrysothrix } & Chrysothrix candelaris (L.) J.R.Laundon* & $\mathrm{Cl}$ \\
\hline & & Chrysothrix xanthina (Vain.) Kalb & So \\
\hline \multirow[t]{2}{*}{ Coenogoniaceae } & \multirow[t]{2}{*}{ Coenogonium } & Coenogonium linkii Ehrenb & $\begin{array}{l}\mathrm{Ch}, \mathrm{Cl}, \mathrm{Mr}, \text { ov, } \\
\text { So, Tv }\end{array}$ \\
\hline & & Leptogium cyanescens (Rabenh.) Körb. & $\mathrm{Cl}, \mathrm{Mr}$, ov, So, Tv \\
\hline \multirow[t]{9}{*}{ Collemataceae } & \multirow[t]{2}{*}{ Leptogium } & Leptogium isidiosellum (Riddle) Sierk & $\mathrm{Cl}, \mathrm{Tv}$ \\
\hline & & Leptogium marginellum (Swartz) S. Gray & $\mathrm{Mr}$, So, Tv \\
\hline & Diorygma & Diorygma poitaei (Fée) Kalb et al.* & $\mathrm{Cl}, \mathrm{Mr}, \mathrm{Ov}, \mathrm{So}, \mathrm{Tv}$ \\
\hline & Dyplolabia & Dyplolabia afzelii (Ach.) A.Massal. & $\mathrm{Ch}, \mathrm{Mr}, \mathrm{Ov}$ \\
\hline & Fissurina & Fissurina dumastii Fée* & $\mathrm{Mr}$ \\
\hline & Glyphis & Glyphis cicatricosa Ach.* & $\mathrm{Cl}, \mathrm{Mr}$ \\
\hline & & Graphis cf. comma (Ach.) Spreng. & Ov \\
\hline & & Graphis dendrogramma $\mathrm{Nyl}$. & $\mathrm{Cl}$ \\
\hline & & Graphis cf. hyphosa Staiger & $\mathrm{Mr}, \mathrm{Ov}$ \\
\hline \multirow{4}{*}{ Graphidaceae } & & Graphis cf. plumierae Vain. & $\mathrm{Cl}, \mathrm{Mr}$ \\
\hline & & Graphis cf. pseudoserpens Chaves, Lücking \& Umaña** & $\mathrm{Ov}$ \\
\hline & & Graphis bifera Zahlbr** & $\mathrm{Ov}$ \\
\hline & & Graphis capillacea Stirt.** & $\mathrm{Tv}$ \\
\hline
\end{tabular}




\section{Steven García-Martínez - Jorge D. Mercado-Gómez}

\begin{tabular}{|c|c|c|c|}
\hline & & Graphis contortuplicata Müll. Arg.** & So \\
\hline & & Graphis copelandii Vain.** & $\mathrm{Mr}$ \\
\hline & & Graphis daintriensis (A. W. Archer) A. W. Archer & $\mathrm{Ov}$ \\
\hline & & Graphis exalbata $\mathrm{Nyl} . * *$ & $\mathrm{Tv}$ \\
\hline & & Graphis ficicola Vain.** & $\mathrm{Cl}$ \\
\hline & & Graphis furcata Fée & $\mathrm{Cl}, \mathrm{Mr}$ \\
\hline & & Graphis hypocrellina Lücking \& Chaves* & $\mathrm{Cl}$ \\
\hline & & Graphis leptocarpa Fée ** & $\mathrm{Tv}$ \\
\hline & & Graphis macella Kremp. & $\mathrm{Cl}$ \\
\hline & Graphis & Graphis rustica Kremp.* & Ov \\
\hline & & Graphis modesta Zahlbr.** & $\mathrm{Ov}, \mathrm{Tv}$ \\
\hline & & Graphis pyrrhocheiloides Zahlbr.** & $\mathrm{Cl}$ \\
\hline & & Graphis rhizicola (Fée) Lücking \& Chávez. & $\mathrm{Cl}$ \\
\hline & & Graphis schiffneri Zahlbr.** & $\mathrm{Cl}$ \\
\hline & & Graphis streblocarpa (Bél.) Nyl.** & $\mathrm{Mr}, \mathrm{So}, \mathrm{TV}$ \\
\hline & & Graphis submarginata Lücking** & $\mathrm{Cl}$ \\
\hline & & Graphis subserpentina $\mathrm{Nyl}^{* *}$ & So, Tv \\
\hline & & Graphis xylophaga (R. C. Harris) Lendemer** & So \\
\hline & & Graphis zonatula Zahlbr.** & So \\
\hline & & $\begin{array}{l}\text { Phaeographis brasiliensis (A. Massal.) Kalb \& Matthes- } \\
\text { Leicht* }\end{array}$ & $\mathrm{Tv}$ \\
\hline & Phaeographis & Phaeographis deightonii C.W. Dodge** & $\mathrm{Mr}$ \\
\hline & & Phaeographis intricans (Nyl.) Staiger & So \\
\hline & & Phaeographis punctiformis (Eschw.) Müll. Arg.** & $\mathrm{Cl}, \mathrm{TV}$ \\
\hline & $\mathrm{C}=\mathrm{ur}$ & Sarcographa labyrinthica (Ach.) Müll.Arg.* & $\mathrm{Cl}$ \\
\hline & Sarcographa & Sarcographa tricosa (Ach.) Müll. Arg. & $\mathrm{Cl}, \mathrm{TV}$ \\
\hline & Sanguinotrema & Sanguinotrema wightii (Taylor) Lücking & $\mathrm{Mr}, \mathrm{Ov}$ \\
\hline & Leucodecton & Leucodecton occultum (Eschw.) A.Frisch & $\mathrm{Cl}, \mathrm{Mr}$ \\
\hline & Ocellularia & Ocellularia erodens (RC Harris) Lücking** & $\mathrm{Ch}, \mathrm{Cl}, \mathrm{Ov}, \mathrm{Tv}$ \\
\hline Incertae sedis & Helminthocarpon & Helminthocarpon leprevostii Fée & $\mathrm{Cl}$, So, Tv \\
\hline & Alyxoria & Alyxoria aff. subrimalis (Nyl.) Cl. Roux \& Poumarat & Tv \\
\hline Lecanographaceae & 7 7 & Zwackhia robusta (Vain.) Ertz & $\mathrm{Ch}$, So \\
\hline & Zwacknia & Zwackhia viridis (Ach.) Poetsch \& Schied. & $\mathrm{Mr}$, So, Tv \\
\hline Lecanoraceae & Lecanora & Lecanora leprosa Fée & $\mathrm{Cl}$ \\
\hline Letrouitiaceae & Letrouitia & Letrouitia domingensis (Pers.) Haf. \& Bellem. & $\mathrm{Cl}, \mathrm{TV}$ \\
\hline & & $\begin{array}{l}\text { Malmidea badimioides (M. Cáceres \& Lücking) M. } \\
\text { Cáceres \& Kalb** }\end{array}$ & $\mathrm{Cl}$ \\
\hline & & Malmidea leptoloma (Müll.Arg.) Kalb \& Lücking & $\mathrm{Cl}, \mathrm{Ov}$, So \\
\hline mid & & Malmidea piperis (Spreng.) Kalb et al. & $\mathrm{Cl}, \mathrm{TV}$ \\
\hline Maımideaceae & Malmidea & $\begin{array}{l}\text { Malmidea rhodopsis (Tuck.) Kalb, Rivas Plata y } \\
\text { Lumbsch** }\end{array}$ & $\mathrm{Cl}, \mathrm{Ov}$ \\
\hline & & Malmidea variabilis Kalb** & So \\
\hline & & Malmidea vinosa (Eschw.) Kalb et al. & $\mathrm{Cl}, \mathrm{Tv}$ \\
\hline
\end{tabular}




\begin{tabular}{|c|c|c|c|}
\hline Megalosporaceae & Megalospora & Megalospora tuberculosa (Fée) Sipman & $\mathrm{Mr}$ \\
\hline Ochrolechiaceae & Varicellaria & $\begin{array}{l}\text { Varicellaria hemisphaerica (Flörke) I. Schmitt \& } \\
\text { Lumbsch* }^{*}\end{array}$ & $\mathrm{Mr}$ \\
\hline \multirow{2}{*}{ Opegraphaceae } & \multirow{2}{*}{ Opegrapha } & Opegrapha dekeselii Ertz & $\mathrm{Cl}$ \\
\hline & & Opegrapha difficilior Nyl. & $\mathrm{Cl}, \mathrm{Ov}$ \\
\hline \multirow{4}{*}{ Parmeliaceae } & \multirow{4}{*}{ Parmotrema } & Parmotrema austrosinense (Zahlbr.) Hale & $\begin{array}{l}\mathrm{Ch}, \mathrm{Cl}, \mathrm{Mr}, \mathrm{Ov} \text {, } \\
\text { So, Tv }\end{array}$ \\
\hline & & Parmotrema cristiferum (Taylor) Hale & Ov \\
\hline & & Parmotrema mesotropum (Müll. Arg.) Hale & $\mathrm{Ch}, \mathrm{Cl}, \mathrm{Tv}$ \\
\hline & & Parmotrema tinctorum (Nyl.) Hale & $\begin{array}{l}\mathrm{Ch}, \mathrm{Cl}, \mathrm{Mr}, \mathrm{Ov} \text {, } \\
\text { So, Tv }\end{array}$ \\
\hline \multirow{2}{*}{ Physciaceae } & \multirow{2}{*}{ Physcia } & Physcia atrostriata Moberg & $\mathrm{Mr}, \mathrm{Ov}$ \\
\hline & & Physcia integrata Nyl.* & $\mathrm{Cl}, \mathrm{Mr}, \mathrm{Tv}$ \\
\hline \multirow{2}{*}{ Pilocarpaceae } & Fellhanera & Fellhanera aff. fuscatula (Müll. Arg.) Vězda & $\mathrm{Cl}$ \\
\hline & \multirow[t]{2}{*}{ Sporopodium } & Sporopodium leprieurii Mont. * & $\mathrm{Mr}$ \\
\hline \multirow{3}{*}{ Pyrenulaceae } & & Pyrenula anomala (Ach.) Vain. & $\mathrm{Mr}$ \\
\hline & \multirow[t]{2}{*}{ Pyrenula } & Pyrenula aspistea (Ach.) Ach. & $\mathrm{Ov}$ \\
\hline & & Pyrenula mamillana (Ach.) Trevis* & $\mathrm{Mr}$ \\
\hline \multirow{5}{*}{ Ramalinaceae } & \multirow[t]{2}{*}{ Bacidia } & Bacidia medialis (Tuck.) Zahlbr. & $\mathrm{Cl}, \mathrm{Mr}, \mathrm{Tv}$ \\
\hline & & Ramalina farinacea (L.) Ach. ** & $\mathrm{Cl}$, So \\
\hline & \multirow[t]{3}{*}{ Ramalina } & Ramalina hypodectodes Nyl. * & $\begin{array}{l}\mathrm{Ch}, \mathrm{Cl}, \mathrm{Mr}, \mathrm{Ov} \text {, } \\
\text { So, Tv }\end{array}$ \\
\hline & & Ramalina sintenisii Müll.Arg.* & $\mathrm{Cl}$ \\
\hline & & Ramalina usnea (L.) R.H.Howe & $\mathrm{Ch}, \mathrm{Cl}, \mathrm{Tv}$ \\
\hline Roccellaceae & Cresponea & Cresponea melanocheloides (Vain.) Egea \& Torrente & $\mathrm{Cl}, \mathrm{Mr}, \mathrm{Tv}$ \\
\hline \multirow[t]{4}{*}{ Strigulaceae } & Strigula & Strigula smaragdula Fr. & $\mathrm{Mr}, \mathrm{So}, \mathrm{Tv}$ \\
\hline & & Astrothelium macrocarpum (Fée) Aptroot \& Lücking & So, Tv \\
\hline & Astrothelium & Astrothelium nitidiusculum (Nyl.) Aptroot \& Lücking & $\mathrm{Cl}$ \\
\hline & & Astrothelium megaspermum (Mont.) Aptroot \& Lücking & $\mathrm{Cl}$ \\
\hline \multirow[t]{4}{*}{ Trypetheliaceae } & Bathelium & Bathelium degenerans (Vain.) R.C.Harris & $\mathrm{Cl}$ \\
\hline & Architrypethelium & $\begin{array}{l}\text { Architrypethelium columbianum (Nyl.) Aptroot \& } \\
\text { Lücking * }\end{array}$ & $\mathrm{Cl}$ \\
\hline & Trypethelium & Trypethelium eluteriae Spreng. & $\mathrm{Mr}, \mathrm{Tv}$ \\
\hline & Nigrovothelium & Nigrovothelium tropicum (Ach.) Lücking et al. & $\mathrm{Mr}$ \\
\hline
\end{tabular}

Las especies con mayor frecuencia en las localidades fueron Coenogonium linkii Ehrenb., Ramalina hypodectodes Nyl., Parmotrema tinctorum (Nyl.) Hale, P. austrosinense (Zahlbr.) Hale, P. mesotropum (Müll. Arg.) Hale y Ramalina usnea (L.) R.H. Howe. Por su parte, otros taxones como Bathelium degenerans (Vain.) R.C. Harris, Chrysothrix xanthina (Vain.) Kalb, Graphis macella Kremp., Graphis pyrrhocheiloides Zahlbr., Herpothallon rubromaculatum G. Thor, Malmidea variabilis Kalb., Varicellaria hemisphaerica (Flörke) I. Schmitt \& Lumbsch, Architrypethelium columbianum (Nyl.)
Aptroot \& Lücking, Astrothelium nitidiusculum (Nyl.) Aptroot \& Lücking, fueron poco frecuentes (un registro cada uno).

\section{DISCUSIÓN}

Con 92 especies, este estudio incrementa las cifras de registros liquénicos para la región Caribe colombiana y el departamento de Sucre. Esta riqueza representa el 42,8 de la diversidad citada para la costa Caribe colombiana y el 5,2\% para Colombia [11-13, 49]. Con respecto a la composición, esta 
es similar a la reportada por otras investigaciones regionales, como es el caso de Rincón, et al. [11], Rincón [12], Lücking, et al. [16]. No obstante, es importante resaltar que la riqueza taxonómica de familias y especies se considera alta si se compara con la registrada para los departamentos de Atlántico, Córdoba y Guajira (215 especies), Cesar (54) y Bolívar (61) en el Caribe colombiano [23-28, 49].

Con respecto a la composición, se pudo establecer que esta es similar a las familias (Graphidaceae, Trypetheliaceae, Roccellaceae, Arthoniaceae, Ramalinaceae, Physciaceae y Thelotremataceae) encontradas por Rincón, et al. [11], Rincón [12], Lücking, et al. [16] en zonas comprendidas entre los departamentos Atlántico, Córdoba, Cesar y la Guajira (Caribe colombiano). La riqueza taxonómica de familias y especies encontrada, se considera alta si se compara con la registrada en otras investigaciones regionales [11-18, 50].

Graphidaceae fue la familia con mayor riqueza de géneros y especies y ordenadas en forma decreciente continuaron Trypetheliaceae, Ramalinaceae, Malmideaceae, Parmeliaceae y Pyrenulaceae. Estos resultados son similares a los hallados por Rincón, et al. [11], Rincón [12], Lücking, et al. [16] en fragmentos de Bs-t en el Caribe colombiano, quienes hallaron una alta riqueza taxonómica de estos taxones. En el caso particular de Graphidaceae, a nivel mundial se ha reportado como la familia con mayor riqueza taxonómica con alrededor de 2161 especies, de esta forma su alta riqueza en los Montes de María solo soportan estos datos [51, 52]. Trypetheliaceae ocupa el segundo lugar de familias de líquenes más ricas en Montes de María. Aptroot, et al. [46] y Aptroot, et al. [46], sugieren que especies de este taxón son muy diversos en bosques secos y sabanas abiertas. En el caso de Roccellaceae, Arthoniaceae, Ramalinaceae, Physciaceae y Pyrenulaceae se ha establecido que presentan una alta riqueza en el sotobosque [53]; en este sentido, los resultados de su alta riqueza en Montes de María pueden relacionarse con el tipo de muestreo, ya que líquenes cortícolas fueron principalmente recolectados, dada la carencia de especímenes en otro tipo sustratos.

Collemataceae y Parmeliaceae, fueron familias importantes de acuerdo a su riqueza en el área de estudio; el registro de estos grupos taxonómicos en Montes de María permite sugerir que estos pueden presentar resistencia a altas temperaturas y habitar ecosistemas más cálidos y, por lo tanto, una posible capacidad de adaptarse a gradientes altitudinales bajos. No obstante, esta hipótesis debe ser puesta a prueba a través de estudios de morfología y anatomía comparativa entre especies que ocurren en bosque seco y bosques húmedos o andinos.

En relación con los géneros, la alta abundancia de Graphis es coincidente con los resultados de Rincón, et al. [11], quienes señalan a este taxón como el mejor representado en planicies del Caribe colombiano. Su alta abundancia puede estar relacionada a su capacidad para colonizar diferentes ecosistemas, llevándolo a ser un grupo cosmopolita, con más de 1200 especies características de micrositios y vegetación abierta en bosques submontanos a montanos bajos en zonas tropicales [52, 54]. Asimismo, Opegrapha, Malmidea, Phaeographis y Parmotrema fueron importantes por su riqueza. En zonas tropicales, Parmotrema y Opegrapha exhiben una gran diversidad y son considerados géneros cosmopolitas que colonizan una amplia gama de sustratos, que incluyen cortezas (ramas y tronco de los árboles), hojas y rocas en vegetación húmeda a seca de elevaciones medianas y en micrositios abiertos $[55,56]$. Malmidea fue otro de los géneros más ricos en el área de estudio. Especies de Malmidea, prefieren microambientes más sombríos y húmedos, condiciones que prevalecen al interior de los bosques en zonas de serranías y montañas de Montes de María. Según García \& Mercado [57], en el área de estudio predominancia de una red hidrográfica y la densa cobertura vegetal las cuales mantienen condiciones microclimáticas de humedad y temperatura. Lo anterior, también podría justificar la presencia de líquenes foliosos y gelatinosos de las familias Parmeliaceae y Collemataceae.

Todas las especies registradas en esta investigación no habían sido reportadas previamente para los fragmentos de bosque seco en los Montes de María, por lo tanto, estos resultados incrementan la riqueza nacional y sugieren que este grupo podría presentar una elevada diversidad característica en este tipo de vegetación. Como nuevos registros de líquenes para Colombia se identificaron: Graphis bifera Zahlbr., G. capillacea Stirt., G. contortuplicata Müll. Arg., G. copelandii Vain., G. exalbata Nyl., G. leptocarpa Fée, G. modesta Zahlbr, G. pyrrhocheiloides Zahlbr, G. submarginata Lücking, G. subserpentina Nyl., G. xylophaga (R. C. Harris) Lendemer, G. zonatula Zahlbr, M. badimioides (M. Cáceres \& Lücking) M. Cáceres \& Kalb, M. rhodopsis (Tuck.) Kalb, Rivas Plata y Lumbsch, M. variabilis Kalb, Ocellularia erodens (RC Harris) Lücking, Phaeographis deightonii C.W. Dodge, 
Phaeographis punctiformis (Eschw.) Müll. Arg y Ramalina farinacea (L.) Ach.

Los resultados de la presente investigación brindan un soporte más sobre la alta diversidad biológica presente en los ecosistemas estacionalmente secos de los Montes de María y, por ende, de los bosques colombianos. Además, se abre una puerta para realizar análisis de ecología de comunidades, con el fin de identificar qué factores han influenciado en la conformación de posibles ensamblajes de especies. Asimismo, aunque en este estudio se presenta el primer listado de especies, también es claro que muchos especímenes aun quedaron sin identificar a nivel de especies, en otras palabras, existe una gran posibilidad que aún existan nuevos taxones para los Montes de María y el país.

\section{AGRADECIMIENTOS}

Al proyecto "Análisis florístico y fitogeográfico sobre relictos de bosque seco tropical en el departamento de Sucre (Colombia)", financiado por la división de Investigaciones de la Universidad de Sucre. A los especialistas Bibiana Moncada y Robert Lücking por las identificaciones taxonómicas recibidas durante el desarrollo de esta investigación.

\section{REFERENCIAS}

\section{Uncategorized References}

[1] N. Chazot, D. L. De-Silva, K. R. Willmott, A. V. L. Freitas, G. Lamas, J. Mallet, et al., "Contrasting patterns of Andean diversification among three diverse clades of Neotropical clearwing butterflies", Ecology and Evolution, vol. 8, pp. 3965-3982, 2018.

[2] J. Aguirre, "Diversidad y riqueza de los líquenes en la región natural Andina o Sistema Cordillerano," in Colombia Diversidad Biótica VI: riqueza y diversidad de musgos y líquenes de Colombia, O. Rangel-Ch, Ed., ed Bogotá: J.O. RANGEL-CH, 2008, pp. 337-382.

[3] J. Aguirre, "Catálogo de los líquenes de Colombia", in Colombia Diversidad Biótica VI: riqueza y diversidad de musgos y líquenes de Colombia, O. Rangel-Ch, Ed., ed Bogotá: J.O. RANGEL-CH, 2008, pp. 401-547.
[4] J. Aguirre-C and J. O. Rangel-Ch, "Amenazas a la conservación de las especies de musgos y líquenes en Colombia -una aproximación inicial-", Caldasia, vol. 29, pp. 235-262, 2007.

[5] M. Medina, "Briófitos y líquenes de los páramos de Moyas y Los Pozos de Aquitania, Boyacá - Colombia", Ciencia en Desarrollo, vol. 2, pp. 17-28, 2006.

[6] M. Pinzón and E. Linares, "Diversidad de líquenes y briofitos en la región subxerofitica de la Herrera, Mosquera (CundinamarcaColombia)", Caldasia, vol. 28, pp. 243257pp, 2006.

[7] B. Moncada, J. Aguirre, and R. Lücking, "Ecogeografía del género Sticta (Ascomycota liquenizados: Lobariaceae) en Colombia", Rev. Biol. Trop. , vol. 62 pp. 257-272, 2014.

[8] B. Moncada and E. Forero, "El género Pseudocyphellaria Vain. (Lobariaceae Lichenized Ascomycetes) en Colombia", Caldasia, vol. 28, pp. 97-215, 2006.

[9] J. Aguirre and K. Avendaño, "Musgos en la Región Caribe", in Colombia Diversidad Biótica VI: riqueza de y diversidad de musgos de Colombia, O. Rangel-Ch, Ed., ed Bogotá: XXX, 2008, pp. 55-59.

[10] R. Álvarez, D. Avendaño, and A. Sanjuan, "La relación entre Peltigera sp. Y Rhizophora mangle en Arroyo de Plata (Bolívar), Caribe colombiano", Luna Azul, vol. 38, pp. 105-121, 2014.

[11] A. Rincón, J. Aguirre, and R. Lücking, "Líquenes Corticícolas en el Caribe Colombiano," Caldasia, vol. 33, pp. 331-347, 2011.

[12] A. Rincón, "Composición de la flora de líquenes corticícolas en el Caribe colombiano", Departamento de Biología, Universidad Nacional de Colombia, Bogotá, Colombia, 2011.

[13] J. Aguirre and K. Avendaño, "Líquenes de la región Caribe", in Colombia Diversidad Biótica VI: riqueza y diversidad de musgos y líquenes de Colombia, O. Rangel-Ch, Ed., ed Bogotá: J.O. RANGEL-CH, 2008, pp. 383-399.

[14] H. Sipman, "Lichens of the Buritica-La Cumbre transect", in La Sierra Nevada de Santa Marta (Colombia) Transecto BuritacaLa Cumbre. Estudios de Ecosistemas Tro- 
pandinos. . vol. 2, T. v. d. H. P. M. Ruíz, Ed., ed, 1984, pp. 185-189.

[15] K. Avendaño and J. Aguirre, "Estudio preliminar de los líquenes de la Serranía del Perijá," in Colombia Diversidad Biótica VIII: Media y baja montaña de la Serrania del Perijá, O. Rangel-Ch, Ed., ed Bogotá: Bogotá, 2009, pp. 223-228.

[16] R. Lücking, B. Moncada, M. Martínez, B. Salgado, M. Celis, O. Rojas, et al., "Lichen diversity in colombian caribbean dry forest remnants", Caldasia vol. 41, pp. 194-214, 2019.

[17] K. Ramírez, "Líquenes de la vertiente noroccidental de la Sierra Nevada de Santa Marta en Colombia," Facultad de Ciencias Básicas, Programa de Biología, Universidad Magdalena, Santa Marta, Colombia, 2018.

[18] R. González, M. López, and P. Silverstone, "Flora terrestre de la isla Malpelo (Colombia), Pacífico Oriental Tropical", Rev. Biol. Trop., vol. 62 pp. 327-336, 2014.

[19] R. Lücking, B. Moncada, M. MartínezHabibe, B. Salgado-Negret, M. Celis, O. Rojas-Zamora, et al., "Lichen diversity in colombian caribbean dry forest remnants," Caldasia, vol. 41, pp. 194-214, 2019.

[20] K. Banda-R, A. Delgado-Salinas, K. G. Dexter, R. Linares-Palomino, A. Oliveira-Filho, D. Prado, et al., "Plant diversity patterns in neotropical dry forests and their conservation implications," Science, vol. 353, pp. 1383-1387, 2016.

[21] C. Pizano, R. Gonzalez, M. Gonzalesz, R. Castro-Lima, N. Rodriguez, A. Idarraga, et al., "Plantas de los bosques secos de Colombia", in El bosque seco tropical en Colombia, C. Pizano and H. García, Eds., ed Bogotá, DC: Instituto de Investigaciones Alexander von Humbolt, 2014, pp. 37-48.

[22] S. Galván-Guevara, B.-D. Gastón, and D. L. O.-V. Jaime, "Determinación de la fragmentación del bosque seco del arroyo Pechelín, Montes de María, Caribe, Colombia," Biota Colombiana, vol. 16, pp. 121-129, 2008.

[23] F. Herazo-Vitola, H. Mendoza-Cifuentes, and J. Mercado-Gómez, "Estructura y composición florística del bosque seco tropical en los Montes de María (Sucre-Colombia)", Ciencia en desarrollo, vol. 8, pp. 79-90, 2017.

[24] J. D. Mercado-Gómez, F. Y. Herazo-Vitola, and M. E. Morales-Puentes, "Phytogeography and Floristic Affinities of Woody Plants in "Los Montes de María", a Tropical Dry Forest Fragment in the Colombian Caribbean", The Botanical Review, vol. 85, pp. 1-19, 2019.

[25] Y. Mercado-Gómez, J. Mercado-Gómez, and C. Giraldo-Sánchez, "Mariposas en un fragmento de bosque seco tropical en Montes de María (Colombia)", Ciencia en desarrollo, vol. 9, pp. 35-45, 2018.

[26] M. A. Sampedro, F. H. Gómez, and D. G. Ballut, "Estado de la vegetación en localidades abandonadas por "desplazamiento", en los montes de María Sucre, Colombia", Recia, vol. 6, pp. 184-193, 2014.

[27] J. Uribe and S. Gradstein, "Estado del conocimiento de la flora de hepáticas de Colombia", Revista de la Academia Colombiana de Ciencias, vol. 23, pp. 315-318, 1999.

[28] S. García, H. Basilio, F. Herazo, J. Mercado, and M. Morales, "Diversidad de briófitos en los Montes de María, Colosó (Sucre, Colombia)", Colombia Forestal, vol. 19, pp. 41-52, 2016.

[29] S. García and J. Mercado, "Diversidad de briófitos en fragmentos de bosque seco tropical,Montes de María, Sucre, Colombia”, Revista Mexicana de Biodiversidad, vol. 88, pp. 824-831, 2017.

[30] M. Aguilera, "Montes de María: Una subregión de economía campesina y empresarial", Documentos de trabajo sobre economía regional. Banco de la República, vol. 195, 2013.

[31] M. Aguilera, "La economía del departamento de Sucre: Ganadería y sector público", vol. 63, ed. Bogotá: Documentos de trabajo sobre economía regional. Banco de la República, 2005, pp. 1-129.

[32] S. Galván, H. Gómez, J. De la Ossa, and A. Fajardo, "Biodiversidad en el área de influencia de la estación primates de Colosó, Sucre, Colombia", Rev. Colombiana cienc. Anim., vol. 1, pp. 98-121, 2009.

[33] Promontes, "Programa de Desarrollo y Paz de los Montes de María", PNUDCORPORACIÓN TERRITORIOS UNIVERSIDAD DE CARTAGENA, Bogotá, 2003. 
[34] G. Halffter, La diversidad biológica de Iberoamérica I, I ed. México: Instituto de Ecología, A.C., 1992.

[35] C. Verbel, "EOT: esquema de ordenamiento territorial diagnóstico integral del territorio (2009). Municipio de Colosó, Departamento de Sucre. (Versión preliminar). Alcaldía de Colosó", ed, 2009.

[36] F. Herazo, J. Mercado, and H. Mendoza, "Estructura y composición florística del bosque seco tropical en los Montes de María (Sucre-Colombia)", Ciencia en Desarrollo, vol. 8, pp. 79-90, 2017.

[37] D. Olascuaga and J. Mercado-Gómez, "Análisis de la vegetación sucesional en un fragmento de bosque seco tropical en ToluviejoSucre (Colombia)", Colombia forestal vol. 19, pp. 23-40, 2016.

[38] R. Lücking, A. W. Archer, and A. Aptroot, "A world-wide key to the genus Graphis (Ostropales: Graphidaceae)", The Lichenologist, vol. 41, pp. 363-452, 2009.

[39] R. Lücking and E. Rivas, "Clave y Guía Ilustrada Para Géneros de Graphidaceae", Glalia, vol. 1, pp. 1-41, 2008.

[40] H. T. Lumbsch, G. B. Feige, and J. A. Elix, "A Revision of the Usnic Acid Containing Taxa Belonging to Lecanora sensu stricto (Lecanorales: Lichenized Ascomycotina)", The Bryologist, vol. 98, pp. 561-577, 1995.

[41] O. Breuss and R. Lücking, "Three new lichen species from Nicaragua, with keys to the known species of Eugeniella and Malmidea", The Lichenologist, vol. 47, pp. 9-20, 2015.

[42] S. HJM. (2005). Mason Hale's key to Parmotrema, revised edition: key to wide-lobed parmelioid species occurring in Tropical America (genera Canomaculina, Parmotrema, Rimelia, Rimeliella). Available: http://www.bgbm.org/ sipman/keys/Neoparmo.htm

[43] M. N. Benatti and M. P. Marcelli, "Physciaceae foliosas do Parque Estadual da Cantareira, estado de São Paulo. III. Espécies do Gênero Physcia”, Rodriguésia, vol. 70, 2019.

[44] A. Aptroot, "A world key to the species of Anthracothecium and Pyrenula", The Lichenologist, vol. 44, pp. 5-53, 2011.

[45] A. Aptroot and R. Lücking, "A revisionary synopsis of the Trypetheliaceae (Ascomycota: Trypetheliales) - ERRATUM", The Lichenologist, vol. 49, pp. 425-425, 2017.
[46] A. Aptroot, M. E. S. CÁCeres, M. K. Johnston, and R. LÜCking, "How diverse is the lichenized fungal family Trypetheliaceae (Ascomycota: Dothideomycetes)? A quantitative prediction of global species richness", The Lichenologist, vol. 48, pp. 983-994, 2016.

[47] R. Lücking, B. P. Hodkinson, and S. D. Leavitt, "The 2016 classification of lichenized fungi in the Ascomycota and Basidiomycota - Approaching one thousand genera", The Bryologist, vol. 119, pp. 361-416, 56, 2017.

[48] M. Chaparro and J. Aguirre, Hongos liquenizados. Bogotá D.C.: Universidad Nacional de Colombia, 2002.

[49] R. Bernal, S. Gradsteins, and M. Celis, Catálogo de plantas y líquenes de Colombia vol. I. Bogotá, Colmbia: Universidad Nacional de Colombia (Sede Bogotá). Facultad de Ciencias. Instituto de Ciencias Naturales, 2016.

[50] J. Aguirre and C. Ruiz, "Composición florística de la brioflora de la Serranía del Perijá (Cesar-Colombia): distribución y ecología", Caldasia vol. 23, pp. 181-201, 2001.

[51] B. Staiger, "Die Flechtenfamilie Graphidaceae. Studien in Richtung einer natürlicheren Gliederung", Bibliotheca Lichenologica vol. 85 , pp. 1-526, 2002.

[52] R. Lücking and E. Rivas, "Clave y guía ilustrada para géneros de Graphidaceae", Glalia vol. 1, pp. 1-41, 2008.

[53] E. Rivas-Plata, R. Lücking, and H. Lumbsch, "When family matters: an analysis of Thelotremataceae (Lichenized Ascomycota: Ostropales) as bioindicators of ecological continuity in tropical forests", Biodivers Conserv vol. 17, pp. 1319-1351, 2008.

[54] R. Lücking, "The taxonomy of the genus Graphis sensu Staiger (Ascomycota: Ostropales: Graphidaceae)", The Lichenologist vol. 41, pp. 319-362, 2009.

[55] D. Ertz, "Revision of the corticolous Opegrapha species from the Paleotropics", Bibliotheca Lichenologica, pp. 1-192, 2008.

[56] J. Chaves, Líquenes tropicales. Costa Rica, 2018.

[57] S. García, "Briófitos y líquenes: una contribución a la biota del bosque seco tropical, subregión Montes de María (Sucre-Colombia)", Departamento de Biología y Química, Universidad de Sucre, Sucre, 2017. 